\title{
Classification of Aircraft Maneuvers for Fault Detection
}

\author{
Nikunj C. Oza, Irem Y. Tumer, Kagan Tumer, and Edward M. Huff \\ Computational Sciences Division \\ NASA Ames Research Center \\ Mail Stop 269-3 \\ Moffett Field, CA 94035-1000 \\ \{o:za, itumer, kagan, huff\}@email arc.nasa.gov
}

\begin{abstract}
Automated fault detection is an increasingly important problem in aircraft maintenance and operation. Standard methods of fault detection assume the availability of either dita produced during all possible fa dity operation modes or a clearly-defined means to determine wh ether the data is a reasonable match to known examples of proper operation. In our domain of fault detection in aircraft, the first assumption is unreasonable and the second is difficult to determine. We envision a system for onlinf fault detection in aircraft, one part of which is a classifier that predicts the maneuver being performed by the aircraft as a function of vibration data and other available data. We explain where this subsystem fits into our envisioned fault detection system as well as experiments showing the promis? of this classification subsystem.
\end{abstract}

\section{Introduction}

A critical aspect 0 : the operation and maintenance of aircraft is detecting problems in their operction when they occur in flight. This allows maintenance and flight crews to fix problems before they become severe and lead to significant aircraft damage or even a crash. Fault detection systems designed for this purpose are becoming a standard requirement in most aircraft. However, most systems are inundated with false alarms, mainly due to an inability to match modeled behavior with real signature; making their reliability questionable in practice [CITE fault detection lit]. Because of the highly critical nature of the aircraft domain application, most fault detection systems are faced with the task of functioning for systems for which fault datia are non-existent. Models are typically used to predict the effect of damage and failures on otherwise healthy (baseline) data $[3,5]$. However, while models are a necessary first start, the modeled system response often doesn't take the operational variability and noise into account, hence resulting in the high 
rates of false aları. Novelty detection is one approach to overcome this problem, addressing the problem of modeling the proper operation of a system and detecting when its operatior. deviates significantly from normal operation $[2,4]$.

In this paper, we present an approach to novelty detection based on in-flight aircraft data. The dita were collected as part of a research effort to understand the sources of variabil.ty present in the actual flight environment, with the purpose of eliminating the high rates of false alarms $[3,5,6]$. 'The fundamental idea is the use of multiple source; of information to predict aspects of system state, such as the maneuver being $p$ rformed, and predicting faults when the system state predictions are incompatible. In this paper, we present several maneuver classifiers. These classifiers take vibration data from various accelerometers and/or other available data as input and predict the maneuver being performed. Multiple subsystems that predict the maneuver may be present in the system. Models of aircraft operation that generate predictions of vibration signatures may also be included in this system. An c verall fault predictor would compare the maneuver predictions from the various subsystems and uses other appropriate data to diagnose whether a fault is present based on these predictions. For exanıle, if the vibration data-based classifier predicts that the helicopter is flying forward at high speed, but other data and/or subsystem: indicate that the aircraft is on the ground, then the probability that a fault is present is high.

In the following, Section 2 discusses the aircraft under study and the data generated from them. We discuss the machine learning methods that we used and the data preparation that we performed in order to use these methods in Section 3. We discuss our experinental results in Section 4. We summarize the results of this paper and discuss ongoing and future work in Section 5.

\section{Aircraft Data}

Data used in this work were collected from two helicopters: an AH1 Cobra and OH58c Kiowa [3]. The data were collected by having two pilots each fly two designated sequences of steady-state maneuvers according to a predetermined test matrix [3]. The test matrix used a modified Latin-square design to counterbalance changes in wind cc nditions, ambient temperature, and fuel depletion. Each of the four flights consisted of an initial period on the ground (Maneuver $G$ ) with the helicopter blades a: flat pitch, a low hover (Maneuver $\mathrm{H}$ ), a sequence of maneuvers drawn from the 12 primary maneuvers, a low hover, and finally a return to ground. Each maneuver was scheduled to last 34 seconds in order to allow a sufficient number of cycles of the main rotor and planetary gear assembly to apply the signal decomposition tech niques used in the previous studies.

Summary matrices were created from the raw data by averaging the data produced during each revolution of the planetary gear. The summarized data consists of 31168 revolutions of data for the AH- 1 and 34144 revolutions of data for the OH58c. Each row, representing one revolution, indicates the maneuver being performed during that revolution as well as columns representing the following 30 quantities: Revolutions per minute of the planetary gear, Torque (four columns: average, standard deviation, skew, and kurtosis), Vibration data from six accelerometers (four columns per accelerometer: root-mean-square, skew. kurtosis, and a binary variable indicating whether signal clipping occurred), Pilot (binary variable). For the AH- 


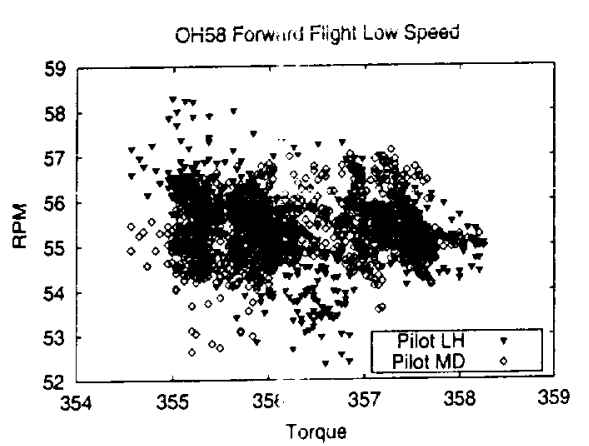

Figure 1: OH58 Naneuver 1 (Forward Figure 2: OH58 Maneuver 4 (Sideward Flight Low Speed)

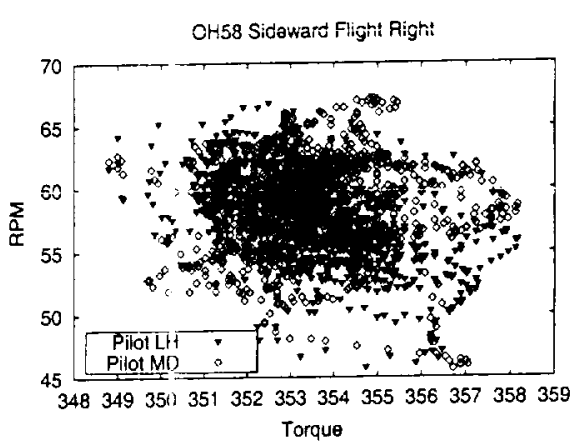

Flight Right)

1, the following aclditional data ( 14 columns) were available for collection from a 1553 bus: Altitude (average and standard deviation), Speed (average and standard deviation), Rate o: climb (average and standard deviation), Heading (average and standard deviation), Bank Angle (average and standard deviation), Pitch (average and standard deviation), Slip (average and standard deviation).

\section{Approach}

Sample data from two selected maneuvers are shown in Figure 2. The highlyvariable nature of the data, as well as differences due to different pilots and different days when the airzraft were flown, are clearly visible, making this a challenging classification problem. To perform the necessary mapping for this problem, we chose multilayer perceptrons (MLPs) with one hidden layer and radial basis function (RBF) networks a: our base classifiers. The first was selected due to its relative ease to use whereas the second for its potential ability to focus on specific "areas" of the feature space [CITE kagan and nikunj's paper]. Furthermore, we constructed ensembles of each type of classifier, as well as ensembles consisting of half MLPs and half RBF netvorks, because ensembles have bren shown to improve upon the performance of the $\mathrm{r}$ constituent or base classifiers, particularly when the correlation among those base classifiers can be kept low $[1,9]$.

We used data sets consisting of all the available features as inputs (44 for the AH1, 30 for the $\mathrm{OH} 58$ ) and one output for each maneuver (14 possible maneuvers in both cases) gathered from the 176 summary matrices. ${ }^{1}$ This resulted in 31168 patterns (revolutions) for the $\mathrm{AH} 1$ and 34144 for the OH58. Both types of classifiers were trained using a randomly-selected two-thirds of the data (21000 examples for the $\mathrm{AH} 1,23000$ for the OH58) and were tested on the remainder for the first set of experiments.

For both data set; and for both types of classifiers, we determined the number of hidden units/ke nels experimentally. For MLPs, we explored hidden layer sizes ranging from 5 to 100 in increments of 5 , and set1led on 25 hidden units for the $\mathrm{AH} 1$ and 65 units for the $\mathrm{OH} 58$. We used a learning rate and momentum term of

\footnotetext{
${ }^{1}$ We linearly transformed all the input features to be in the $[-2,2]$ range.
} 
Table 1: Sample confusion matrix for OH58 (MLP).

\begin{tabular}{|c|c|c|c|c|c|c|c|c|c|c|c|c|c|c|}
\hline \multirow[b]{2}{*}{ Class } & \multicolumn{14}{|c|}{ Classification } \\
\hline & 1 & 2 & 3 & 4 & 5 & 6 & 7 & & 9 & 10 & $\pi$ & 12 & 13 & 14 \\
\hline I & 693 & $\sigma$ & & 6 & $\overline{79}$ & $\overline{0}$ & $\overline{0}$ & 0 & $\overline{0}$ & 0 & 0 & $\overline{0}$ & 0 & 0 \\
\hline 2 & 0 & 679 & o & 0 & 0 & 0 & 0 & 0 & $\hat{0}$ & 0 & 0 & 0 & 47 & 0 \\
\hline 3 & 55 & 1 & 568 & 64 & 31 & 6 & 0 & 11 & 9 & 1 & 11 & 7 & 0 & 3 \\
\hline 4 & 26 & $\bar{\sigma}$ & 13 & 691 & 15 & 0 & $\bar{a}$ & 3 & 0 & 0 & 0 & 2 & 0 & 1 \\
\hline 5 & 196 & 0 & 68 & 41 & 412 & 0 & 0 & 0 & 0 & 0 & 2 & 16 & 0 & 0 \\
\hline 6 & 0 & $\partial$ & 0 & 0 & 0 & 719 & 0 & 0 & 0 & 0 & 0 & $\overline{0}$ & 0 & 0 \\
\hline 7 & 0 & 0 & 0 & 0 & 0 & 0 & 1079 & 0 & 0 & 0 & 0 & 0 & 0 & 0 \\
\hline 8 & $\overline{0}$ & 9 & 22 & $\overline{16}$ & 0 & 0 & $\overline{0}$ & 748 & 177 & 97 & 11 & 6 & 3 & 0 \\
\hline 9 & 0 & 1 & 1 & 6 & 0 & 0 & 0 & 172 & 381 & 162 & 4 & 7 & 6 & 0 \\
\hline 10 & 0 & 4 & 1 & 6 & 0 & 0 & 0 & 186 & 170 & 376 & 0 & 8 & 13 & 0 \\
\hline 11 & 4 & 0 & 15 & 4 & 3 & 0 & 0 & 2 & 1 & 0 & 494 & 217 & 0 & 0 \\
\hline 12 & 3 & 0 & 7 & 6 & 4 & 0 & 0 & 2 & 1 & 0 & 200 & 531 & 0 & 0 \\
\hline 13 & 0 & 63 & $-\overline{0}$ & 0 & 0 & 0 & 0 & 4 & 1 & 0 & 0 & 0 & 712 & 0 \\
\hline 14 & 0 & 0 & 0 & 0 & 0 & 0 & 0 & $\overline{0}$ & 0 & $\sigma$ & 0 & 0 & 0 & 685 \\
\hline
\end{tabular}

0.2 , and we trained for 100 epochs. The performance of both types of classifiers was fairly insensitive to both the hidden unit size/number of kernels and learning parameters. We created 280 MLPs for each helicopter, and we report results as averages over these 280 runs. These 280 MLPs were given different random initial weights before training, but were trained using the same training sets.

For the RBF networks, we used 100 centers for the $0 \mathrm{H} 58$ data and determined each kernel's center anc width using the nearest 300 patterns. ${ }^{2}$ For the AH1 data, we used 55 kernels with the centers and widths determi:ned by the nearest 500 patterns. For each helicopte: we created 100 RBF networks, each of which had a different set of centers, and report results as averages over these 100 runs. ${ }^{3}$

For both data setc and classifiers, we used simple averaging ensembles. Though simple to apply, such ensembles perform remarkably well on a variety of data sets [1, $7,8]$. We experimented with ensembles consisting of 2 to 100 base classifiers for our MLP and MLP/RIFF ensembles, and 2 to 50 base clissifiers for our RBF ensembles, although performance improvements after 10 base classifiers were marginal. These ensembles consisted of random samples drawn from the $280 \mathrm{MLPs}$ and $100 \mathrm{RBF}$ networks that we created for our single-network ixperiments. For each size of ensemble, we drew 20 random samples and report the results as averages over these runs.

In addition, we calculated the confusion matrix of every classifier we created. Entry $(i, j)$ of the confurion matrix of a classifier states the number of times that an example of class $i$ is classified as class $j$. In examining the confusion matrices of our classifiers (see Table 1 for an example of a confusion matrix - entry $(1,1)$ is in the upper left corner), we noticed that particular maneuvers were continually being confused with one another. In particular, the three hover maneuvers (8Hover, 9-Hover Tum Left, and 10-Hover Turn Right) were frequently confused with one another and the two coordinated turns (11-Coordinated Turn Left) and (12Coordinated Turn Right) were also frequently confused (the counts associated with these errors are shown in bold in Table 1. These sets of maneuvers are similar enough to one another that misclassifications within these groups are unlikely to imply the

\footnotetext{
${ }^{2}$ That is, for each center, the 300 training cases closest to it in Euclidian distance were used to determine it:; radius. Therefore, the radius increases with the number of points.

${ }^{3}$ Due to the large computation time needed to obtain the centers and widths of the kernels on such large data sets, we only used 100 RBFs as opposed to 280 MLPs.
} 
Table 2 OH58c and AH1 Single Revolution Test Set Results.

\begin{tabular}{|c|c|c|c|c|c|}
\hline \multirow[b]{2}{*}{$\begin{array}{l}\text { Base } \\
\text { Type }\end{array}$} & \multirow[b]{2}{*}{$\mathrm{N}$} & \multicolumn{2}{|c|}{ OH58 Results } & \multicolumn{2}{|c|}{ AH1 Results } \\
\hline & & $\begin{array}{l}\text { Single } \\
\text { Rev }\end{array}$ & $\begin{array}{l}\text { Single Rev } \\
\text { Confusion } \\
\end{array}$ & $\begin{array}{c}\text { Single } \\
\text { Rev }\end{array}$ & $\begin{array}{l}\text { Single Kev } \\
\text { Confusion }\end{array}$ \\
\hline \multirow{4}{*}{ MLP } & 1 & $\overline { 8 1 } \longdiv { . 5 3 3 \pm 0 . 1 1 0 }$ & $93.098 \pm 0.073$ & $96.161 \pm 0.138$ & $98.643 \pm 0.094$ \\
\hline & 4 & $83.114 \pm 0.063$ & $94.307 \pm 0.038$ & $97.747 \pm 0.071$ & $99.583 \pm 0.064$ \\
\hline & 10 & $8: 3.578 \pm 0.047$ & $94.470 \pm 0.025$ & $98.089 \pm 0.042$ & $99.737 \pm 0.041$ \\
\hline & 100 & $83.960 \pm 0.018$ & $94.683 \pm 0.010$ & $98.225 \pm 0.008$ & $99.818 \pm 0.003$ \\
\hline \multirow{4}{*}{ RBF } & 1 & $7.650 \pm 0.142$ & $90.860 \pm 0.104$ & $95.811 \pm 0.098$ & $99.106 \pm 0.060$ \\
\hline & 4 & $78.408 \pm 0.089$ & $91.384 \pm 0.052$ & $96.272 \pm 0.032$ & $99.390 \pm 0.035$ \\
\hline & 10 & $78.550 \pm 0.039$ & $91.607 \pm 0.027$ & $96.441 \pm 0.021$ & $99.472 \pm 0.013$ \\
\hline & 50 & $73.729 \pm 0.018$ & $91.638 \pm 0.011$ & $96.438 \pm 0.009$ & $99.493 \pm 0.000$ \\
\hline \multirow{3}{*}{$\begin{array}{l}\text { MLP/ } \\
\text { RBF }\end{array}$} & 2 & $8 . .851 \pm 0.087$ & $93.548 \pm 0.053$ & $97.392 \pm 0.069$ & $99.515 \pm 0.053$ \\
\hline & 4 & $82.124 \pm 0.084$ & $94.097 \pm 0.047$ & $07809+0.019$ & $99.764+0.011$ \\
\hline & 100 & $8.398 \pm 0.023$ & $94.548 \pm 0.014$ & $97.989 \pm 0.007$ & $99.791 \pm 0.003$ \\
\hline
\end{tabular}

presence of faults. Therefore, for our second set of experiments, we recalculated the classification acculacies after consolidating these maneuvers (e.g., all three hovers into one maneuver and both left and right turns into one maneuver).

Finally, we used the knowledge that a helicopter needs some time to change maneuvers. That is, two sequentially close patterns are unlikely to come from different maneuvers. To of tain results that use this "prior" knowledge, we tested on sequences of revolutions by averaging the classifiers' outputs on a window of examples surrounding the current one. In one set of experiments, we averaged over windows of size 17 ( 8 revolutions before the current one, the current one, and 8 revolutions after the current (ne) which corresponds to about three seconds. Note that, because the initial training and test sets were randomly chosen from this sequence, this averaging cou d not be performed on the test set alone. Instead it was performed on the full data set for both helicopters. To allow meaningful comparisons of these results, wt also computed the "full set error" (training and test errors) on the original, segmented data and these results are presented in Tables $3,4 .^{4}$

\section{Results}

In this section we describe the experimental results that we have obtained so far. In Table 2, the column marked "Single Rev" shows the results of running individual networks and ensembles of various sizes on the summary matrices randomly split into training and test sets. We only present result; for some of the ensembles we constructed due to space limitations and because the ensembles exhibited relatively small gains beyond 10 base models. $N$ is the number of base models used for the classification. NULPs and ensembles of MLPs outperform RBFs and ensembles of RBFs consisten ly. The ensembles of MLPs improve upon single MLPs to a greater extent than ensembles of RBF networks do upon single networks, indicating that the MLPs arf more diverse than the RBF networks. Mixed ensembles have

\footnotetext{
${ }^{4}$ We performed this windowed averaging as though the entire data were collected over a single flight. However, it was in fact collected in stages, meaning that there are no transitions between maneuvers. We show these results to demonstrate the applicability of this method to sequential data obtained in actual flight after training the network on "static" single revoli tion patterns.
} 
Table 3: OH58: Single Revolution and Windowing Results on Full Data Set.

\begin{tabular}{|c|c|c|c|c|c|}
\hline $\begin{array}{l}\text { Base } \\
\text { Type }\end{array}$ & $\mathrm{N}$ & $\begin{array}{l}\text { Single } \\
\text { Rev }\end{array}$ & $\begin{array}{c}\text { Single Rev } \\
\text { Consolidated }\end{array}$ & $\begin{array}{l}\text { Window } \\
\text { of } 17\end{array}$ & $\begin{array}{l}\text { Window of } \\
17 \text { Consolidated }\end{array}$ \\
\hline \multirow{4}{*}{ MLP } & 1 & $82724 \pm 0.121$ & $94.067 \pm 0.049$ & $8 9 \longdiv { 8 1 3 \pm 0 . 1 9 1 }$ & $96.799 \pm 0.142$ \\
\hline & 4 & $85466 \pm 0.073$ & $95.020 \pm 0.034$ & $91.287 \pm 0.130$ & $96.956 \pm 0.043$ \\
\hline & 10 & $86035 \pm 0.050$ & $95.243 \pm 0.034$ & $91.550 \pm 0.081$ & $97.006 \pm 0.044$ \\
\hline & 100 & $86414 \pm 0.015$ & $95.420 \pm 0.007$ & $91.621 \pm 0.022$ & $97.067 \pm 0.008$ \\
\hline \multirow{4}{*}{ RBF } & 7 & $79484 \pm 0.053$ & $91.313 \pm 0.099$ & $8.6 .670 \pm 0.212$ & $95.008 \pm 0.115$ \\
\hline & 4 & $27 \pm 0.094$ & $91.786 \pm 0.045$ & $8.4 .739 \pm 0.131$ & $95.026 \pm 0.058$ \\
\hline & 10 & $79297 \pm 0.047$ & $91.975 \pm 0.020$ & $84.977 \pm 0.070$ & $95.232 \pm 0.045$ \\
\hline & 50 & $79460 \pm 0.014$ & $92.014 \pm 0.008$ & $85.086 \pm 0.021$ & $95.103 \pm 0.017$ \\
\hline \multirow{3}{*}{$\begin{array}{l}\text { MLP/ } \\
\text { RBF }\end{array}$} & 2 & $83740 \pm 0.093$ & $94.212 \pm 0.063$ & $89.935 \pm 0.163$ & $96.508 \pm 0.084$ \\
\hline & & $84710 \pm 0.075$ & $94.748 \pm 0.048$ & $90.493 \pm 0.125$ & $96.779 \pm 0.069$ \\
\hline & 10 & $85280 \pm 0.038$ & $95.012 \pm 0.030$ & $\begin{array}{l}90.755 \pm 0.068 \\
90.838 \pm 0.029\end{array}$ & $\begin{array}{l}96.869 \pm 0.043 \\
96.822 \pm 0.014\end{array}$ \\
\hline
\end{tabular}

performances superior to the pure-MLP for smal: numbers of base models, but have worse perfornances for larger numbers of models. Mixed ensembles perform better than pure-RBF ensembles for all numbers of base models. In the smaller ensembles, the diversity provided by including RBF networks helped relative to pure-MLP ensembles. However, in the larger ensembles, replacing half the MLPs with RBFs degrades performance- the RBFs are different from the MLPs but not different enough from each other to warrant havin:s such a large number of them. Note that the column marked "Single Rev Confusion" shows the single revolution results after allow ng for confusions among the hover maneuvers and among the coordinated turns. As expected, the performances improved dramatically.

Table 3 shows the results of performing the windowed averaging described in the previous section in the column marked "Window of 17 ." The column "Window of 17 Confusion" givts the results allowing for the confusions mentioned earlier. The columns marked "Single Rev" and "Single Rev Confusion" are the average of the training and test errors, weighted by their sizes. We can clearly see the benefits of this windowed averaging, which serves to smooth out some of the noise present in the data.

Table 4: AH1 Single Revolution and Windowing Results on Full Data Set.

\begin{tabular}{|c|c|c|c||c|c|}
\hline $\begin{array}{c}\text { Base } \\
\text { Type }\end{array}$ & $\mathrm{N}$ & $\begin{array}{c}\text { Single } \\
\text { Rev }\end{array}$ & $\begin{array}{c}\text { Single Rev } \\
\text { Confusion }\end{array}$ & $\begin{array}{c}\text { Window } \\
\text { of } 17\end{array}$ & $\begin{array}{c}\text { Window of } \\
17 \text { Confusion }\end{array}$ \\
\hline \hline \multirow{3}{*}{ MLP } & 1 & $96.567 \pm 0.115$ & $98.789 \pm 0.081$ & $97.821 \pm 0.111$ & $98.744 \pm 0.086$ \\
& 10 & $98.007 \pm 0.064$ & $99.561 \pm 0.060$ & $98.933 \pm 0.080$ & $99.374 \pm 0.082$ \\
& 100 & $98.438 \pm 0.041$ & $99.769 \pm 0.042$ & $99.179 \pm 0.040$ & $99.621 \pm 0.039$ \\
& 1 & $96.023 \pm 0.093$ & $99.852 \pm 0.003$ & $99.268 \pm 0.004$ & $99.700 \pm 0.002$ \\
\hline \multirow{2}{*}{ RBF } & 4 & $96.480 \pm 0.031$ & $99.209 \pm 0.051$ & $97.120 \pm 0.114$ & $98.931 \pm 0.064$ \\
& 10 & $96.638 \pm 0.015$ & $99.535 \pm 0.011$ & $97.635 \pm 0.044$ & $99.141 \pm 0.023$ \\
& 50 & $96.649 \pm 0.008$ & $99.558 \pm 0.005$ & $97.624 \pm 0.005$ & $99.187 \pm 0.011$ \\
& 2 & $97.664 \pm 0.059$ & $99.611 \pm 0.045$ & $93.564 \pm 0.062$ & $99.327 \pm 0.053$ \\
MLP/ & 4 & $97.957 \pm 0.052$ & $99.699 \pm 0.046$ & $98.725 \pm 0.056$ & $99.390 \pm 0.055$ \\
RBF & 10 & $98.092 \pm 0.017$ & $99.796 \pm 0.010$ & $98.818 \pm 0.021$ & $99.516 \pm 0.012$ \\
& 100 & $98.144 \pm 0.014$ & $99.810 \pm 0.008$ & $98.852 \pm 0.006$ & $99.546 \pm 0.003$ \\
\hline
\end{tabular}

Table 4 shows the analogous results for the AH1 helicopter. The performances are substantially better here than for the $\mathrm{OH} 58$. We expected this because the AH1 
Table 5: AH1 Bus and Non-Eus Results

\begin{tabular}{|c|c|c|c|c|}
\hline Inputs & $\begin{array}{l}\text { Single } \\
\text { Rev }\end{array}$ & $\begin{array}{l}\text { Single Rev } \\
\text { Confusion }\end{array}$ & $\begin{array}{l}\text { Window } \\
\text { of } 17\end{array}$ & $\begin{array}{l}\text { Window of } \\
17 \text { Confusion }\end{array}$ \\
\hline Bus & $90.180 \pm 0.110$ & $95.871 \pm 0.091$ & $91209 \pm 0.126$ & $96.027 \pm 0.086$ \\
\hline Non-Bus & $87.884 \pm 0.228$ & $93.731 \pm 0.171$ & $92913 \pm 0.355$ & $96.110 \pm 0.236$ \\
\hline$P($ agree $)$ & $79.523 \pm 0.247$ & $90.063 \pm 0.202$ & $85609 \pm 0.320$ & $93.393 \pm 0.247$ \\
\hline
\end{tabular}

is a heavier helicopter, so it is less affected by conditions that tend to introduce noise such as wind changes. Just as with the $\mathrm{OH} 58$, on the AH1, the mixed ensembles outperform the pure ensembles for small numbers of base models but perform worse than the MLP ensembles for larger numbers of base models. Once again, we can see that ensembles of MLPs outperform single MLPs to a greater extent than ensembles of RBFs outperform single RBFs, so the RBFs are not as different from one another. Because of this, it does not hel] to add large numbers of RBF networks to an MCP ensemble. Note that the same sets of maneuvers that were frequently confused on the OH58 were confused on the AH1. Taking this confusion into account boosted performance significantly. The windowed averaging approach did not always yield improvement when allowing for the maneuver confusions, but helped when classifying across the full set of maneuvers. However, in all cases when windowed averaging did not help, the classifier performance was at least $98.93 \%$, so there was very little room for improvement.

\section{Discussion}

In this paper, we fresented an approach to fault detection that contains a subsystem to classify an operating aircraft into one of several states. More specifically, the proposed systen determines the maneuver being performed by an aircraft as a function of vibration data and any other available data. Through experiments with two helicopters, we demonstrated that the system is able to determine the maneuver being performed with good reliability (at least $95 \%$ when allowing for confusions among very similar system states and smoothing by combining predictions from short sequences of data). The initial results show great promise in classifying the correct maneuver with high certainty. Future work will involve applying this approach to "free-flicht data", where the maneuvers are not static or steady-state, and transitions between maneuvers exist.

The results presented in this paper address the maneuver classification portion of the online fault detection system envisioned in this jesearch. To address the overall novelty detection froblem, future work will involve experiments to determine the probabilities of agr zement between different classification results, to detect possible faults when there is a mismatch. For example, for the AH1 helicopter, we have data from a 1553 bus as described in Section 2. We trained some classifiers using just the bus data as inputs and other classifiers using all except the bus data. Table 5 shows just the results of raining 20 single MLPs on these data using the same network topology as for the other MLPs trained on all the AH1 data. They performed much worse than the single MLPs trained with all the inputs presented at once. The last line in the table indicates the percentage of maneuvers for which the two types of classifiers agreed. 
Recall from Section 1 that we would like classifier disagreement to indicate the presence of a fault; therefore, we would like these agreement probabilities to be much higher. However, we hypothesize that we can use the bus data in a much simpler way. For example, if the vibration data-based classifier predicts that the aircraft is performing a high-speed forward fight, but the bus data indicates that airspecd is near zero, then the probability of a fault is high. We do not necessarily need a system that returns the maneuver as a function of all the variables that constitute the bus data. In this example, we merely need to know that a near-zero airspeed is inconsistent with i high-speed forward flight. We flan to perform a detailed study of the collected bus data so that we may construct simple classifiers representing knowledge of the type just mentioned and use them to find inconsistencies such as what we just described. We are confident that using the different types of system models, metrics, and classifiers mentioned in this paper, we can obtain a reliable fault detector.

\section{References}

[1] L. Breiman. B.xgging predictors. Machine Learning, 24(2):123-140, 1996.

[2] Paul Hayton, Hernhard Schölkopf, Linel Tarassınko, and Paul Anusiz. Support vector novelty detection applied to jet engine vibration spectra. In Todd $\mathrm{K}$. Leen, Thomas G. Dietterich, and Volker Tresp, editors, Advances in Neural Information Processing Systems-13, pages 946-952. Morgan Kaufmann, 2002.

[3] Edward M. Huff, Irem Y. Tumer, Eric Barszcz, Mark Dzwonczyk, and James McNames. Analysis of maneuvering effects on transmission vibration patterns in an AH-1 cotra helicopter. Journal of the American Helicopter Society, 2002.

[4] Nathalie Japkowicz, Catherine Myers, and Mark Gluck. A novelty detection approach to classification. In Proceedings of the 14 th International Joint Conference on Artificial Intelligence, pages 518-523, Montreal, Canada, 1995. Morgan Kaufmann.

[5] D.A. McAdam: and I.Y. Tumer. Towards failure modeling in complex dynamic systems: impact of design and manufacturing variations. In ASME Design for Manufacturing Conference, volume DETC2002/DFM-34161, September 2002.

[6] I.Y. Tumer anc E.M. Huff. On the effects of production and maintenance variations on machinery performance. Journal of Quality in Maintenance Engineering, To appear. 2002.

[7] K. Tumer and J. Ghosh. Analysis of decision troundaries in linearly combined neural classifiers. Pattern Recognition, 29(2):341-348, February 1996.

[8] K. Tumer and J. Ghosh. Error correlation and error reduction in ensemble classifiers. Connection Science, Special Issue on Combining Artificial Neural Networks: Ensemble Approaches, 8(3 \& 4):385-404, 1996.

[9] D. H. Wolpert. Stacked generalization. Neural Networks, 5:241-259, 1992. 